\title{
Entrelacs
}

Cinéma et audiovisuel

HS | 2008

Séries télé in/out

\section{Composition générique}

Stéphane Massa-Bidal

\section{OpenEdition}

Journals

Édition électronique

URL : http://journals.openedition.org/entrelacs/258

DOI : $10.4000 /$ entrelacs.258

ISSN : 2261-5482

Éditeur

Éditions Téraèdre

Édition imprimée

Date de publication : 7 février 2008

ISSN : 1266-7188

Référence électronique

Stéphane Massa-Bidal, « Composition générique », Entrelacs [En ligne], HS | 2008, mis en ligne le 01 août 2012, consulté le 21 décembre 2020. URL : http://journals.openedition.org/entrelacs/258 ; DOI : https://doi.org/10.4000/entrelacs.258

Ce document a été généré automatiquement le 21 décembre 2020.

Tous droits réservés 


\title{
Composition générique
}

\author{
Stéphane Massa-Bidal
}

1 Gros plan sur son passeport, un arc électrique, une expérience physico-chimique, le voilà qui trafique une boussole, redirige un rayon laser, bricole un levier de vitesse avec des sangles, place un pain de plastique, réalise un branchement électrique, plie et déplie son couteau suisse... Vous avez deviné de qui il s'agit? Son nom s'inscrit dans une explosion: Mac Gyver; sur les plans suivants, notre héros se révèle atteint d'une grave hypertrophie de compétences de bricoleur-cascadeur...

2 Pratique sociale de l'entour des séries, ${ }^{1}$ le générique est aisément connu et reconnu par son aspect musical. Cependant, au-delà de la dimension musicale, quels sont les ingrédients et recettes? Présentation des héros, des lieux, mise en récit ou non, signes plastiques, typographie, musique, paroles, placement à l'intérieur de la série, au début et à la fin de l'épisode, variation au fil des saisons... objet syncrétique, il fonde par sa relative autonomie par rapport à la série, un discours sur celle-ci et d'autre part, par son contexte de production, il fonde un discours de la série. À partir d'une approche et d'une délimitation du territoire générique (la plupart des génériques cités ici sont visualisables sur les sites http://www.youtube.com ou http://www.dailymotion.com), nous analyserons les dernières innovations de ce dernier. Si le générique est à la série ce que le plus petit commun multiple (PPCM) est à l'arithmétique, il se retrouve obstinément sur tous les épisodes et saisons, et ambitionne la " loi des séries "; armés d'un corpus varié et diachronique, nous observerons le rapport série/générique. Enfin, nous établirons une typologie des génériques, des règles de construction et de placement à l'intérieur de l'épisode.

\section{Tour du propriétaire et délimitations}

3 Un épisode de série condense un syntagme composé de paratextes : générique de début, de fin, du contenu de l'épisode, du rappel des éléments narratifs, des mentions de production, logos/séquences, de la(les) musique(s) et d'une typographie spécifique (e.g. : le caractère du slash dans Nip/Tuck est motivé par l'incision des chirurgiens). Du point de 
vue pragmatique, un générique se reconnaît par trois aspects: son "genre ", son placement dans l'épisode et sa répétition au cours de la saison.

4 Commençons par la fin: un générique de fin détaille la production, l'ensemble du personnel technique et des mentions musicales. Il est souvent composé d'un fond uni et sombre d'une typographie de couleur claire ; la musique de la série est rejouée (la plupart du temps) une seconde fois, et présente les mentions obligatoires, techniques et acteurs secondaires. Certaines séries (Mac Gyver, N.C.I.S.), créent des génériques de fin ad hoc en reprenant des images de l'épisode en les montant dans un diaporama-souvenir de l'épisode. Cette fonction-résumé pouvant avoir un effet de mémoire pour la suite de la série, et imprimer la mémoire du spectateur. Arabesque joue la paresse et repasse la musique sur un plan fixe.

D'une manière générale, le générique de fin fluctue en fonction du casting ou de la production, alors que celui du début est invariant. Le générique de début est plus dynamique et plus court (de 10 secondes à $1 \mathrm{~min}$. 30) que celui de fin et présente le plus souvent le(s) acteur(s) et précise le(s) personnage(s) principaux qu'il(s) incarne(nt) (Sex and the city se focalise sur son personnage central). À la suite de ce générique, un ensemble de mentions de production sont intégrées, dans le début de l'épisode.

Ainsi, le générique de début a une valeur générale, par sa quasi-invariance, par sa reproduction du même, par sa distance par rapport à l'épisode alors que celui de fin a une valeur restreinte, cantonnée à l'épisode; le générique de fin constitue un discours sur l'épisode et celui du début un discours sur la série.

Tableau 1 : oppositions entre le générique de début et de fin

\begin{tabular}{|l|l|l|}
\hline Thématique & Générique de début & Générique de fin \\
\hline Variabilité & invariant & Variant \\
\hline Tempo & Dynamique & Lent \\
\hline Mentions & Personnages principaux & Production entière \\
\hline Longueur & Court & Long \\
\hline Rapport générique/genre & En rapport avec le genre & Sans rapport avec le genre \\
\hline Visée & Partielle & Exhaustive \\
\hline Visibilité & Diffusé & « Zappé » \\
\hline Identité & Forte identité visuelle & Faible identité visuelle \\
\hline Narration & Fortement scénarisé & Faiblement scénarisé \\
\hline Valeur & Introductive & Finale \\
\hline Unicité & Répété & Unique \\
\hline Couleur & Plutôt clair & Plutôt foncé \\
\hline
\end{tabular}




\begin{tabular}{|l|l|l|}
\hline Visée & Série & Épisode \\
\hline
\end{tabular}

5 Si David Vincent les a vus, les voila qui sèment le trouble, et le générique des Envahisseurs fait figure d'O.V.N.I. énonciatif : une voix-off introduit théâtralement les acteurs, glissement de l'écrit vers l'oral d'une part et installation d'une présence énonciatrice d'autre part. Cette excentricité ira jusqu'à nous présenter les interprètes de l'épisode sans leur rôle, mais en mentionnant le nom de l'épisode. Ces incartades soulignant le rapport particulier réel/fiction, comme si l'on attirait l'attention du spectateur vers les conditions d'interprétation du genre fantastique ${ }^{2}$. Spatial toujours, Cosmos 1999 fait place à des mini-scènes spectaculaires avec la mention doublement répétée " dans cet épisode ", à la manière d'une table des matières, un teasing intégré au générique.

\section{Matériaux et narrations}

Quel type de rapport de construction intertextuelle entretient un générique par rapport à la série? Sur le plan des matériaux, nous avons trois types de génériques: création complète, reprise d'éléments et mixité des deux premiers schématisés sur le tableau suivant:

Typologies de génériques sur le plan de l'expression

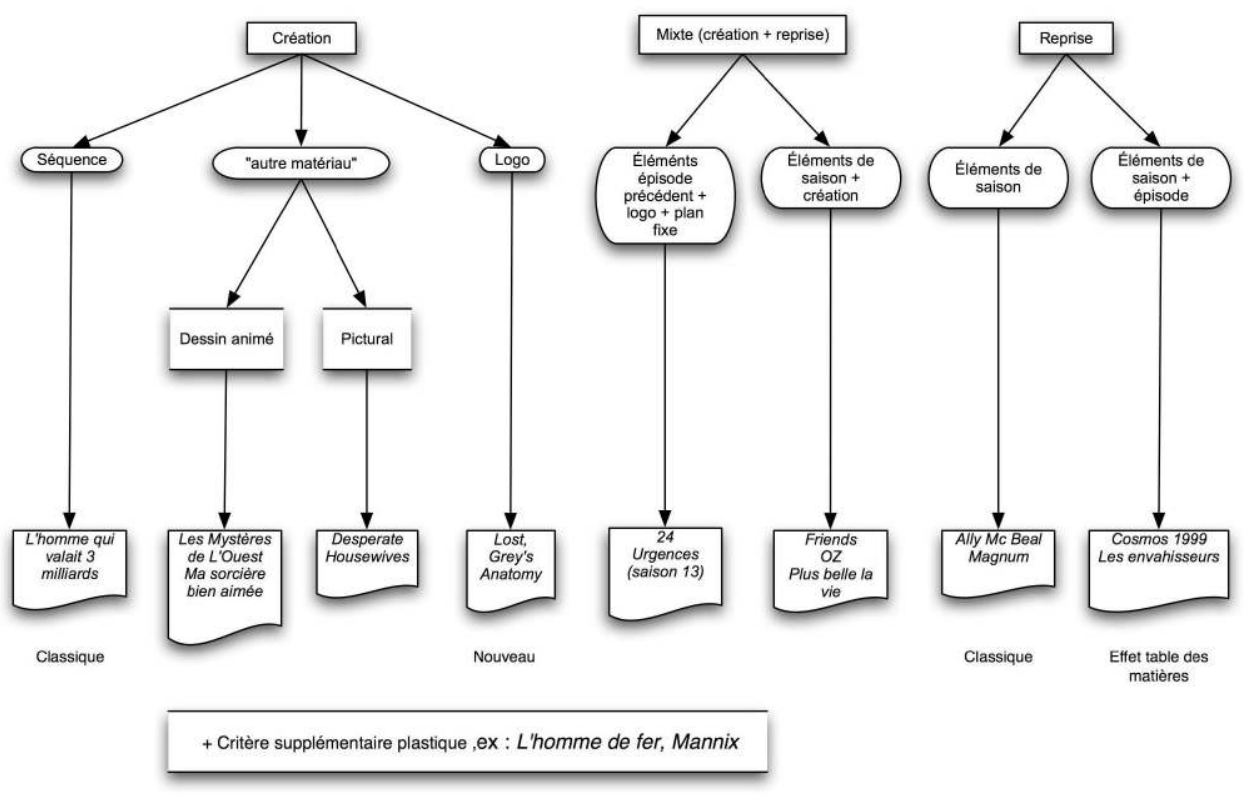

7 Ajoutons un critère plastique sur le plan de l'expression: les génériques des années cinquante/soixante utilisaient les mêmes effets graphiques que ceux utilisés au cinéma, apportant une scansion de la séquence en imposant un rythme soutenu et des images collapsées. L'effet graphique servant à la narration avec un "effet poursuite " pour Cannon, et surtout hypnotique pour le spectateur, utilisant des principes de l'op art ou art cinétique. Tout se passe comme si l'on faisait jouer au générique la fonction d'un signal perceptif ${ }^{3}$ (alternances de couleurs, de noir et de plans rapides). Dans un autre cas, si Médium fait référence aux tests de Rorschach, le générique intertextuel de Desperate 
Housewives est monté à la manière d'un collage avec neuf citations d'œuvres appartenant à l'art ou à la publicitét. Ce type de générique utilise des matériaux différents, voire même des dessins animés ${ }^{5}$, installant une connotation ludique et une distance avec la série. Aussi, d'un point de vue énonciatif, le générique est un acte énonciatif méta-narratif, où un énonciateur « supervise » l'énoncé de l'épisode, pour le communiquer (ou le signifier) à un énonciataire qui sera ensuite le "narrataire » de l'épisode; le tempo syncopé participant à l'acte énonciatif et identitaire de la série.

\section{Nouvelles tendances}

\section{Le mini, nouvelle tendance}

8 Alors qu'un générique dure environ de trente à quatre-vingt-dix secondes, des séries comme Lost ou le mini générique-logo de Grey's anatom ${ }^{6}$. Économie de temps d'antenne ou véritable stratégie identitaire de production de générique ? Effet de genre?

L'utilisation du logo n'est pas récente: le générique du Saint est à rapprocher de ce procédé. Cet aspect trouve sa raison dans le processus de communication et de pratique sociale. Dans un contexte de consommation exacerbée : émetteur et destinataire règlent leurs pratiques : fans de série, programmation régulière des épisodes, téléchargement, visionnage de DVD..., et reprogrammation, cette économie d'image trouve sa raison dans une économie de temps d'antenne et de production ${ }^{7}$, et à l'autre bout de la chaîne, le spectateur reste "concentré » et disponible, évitant le zapping ou la «coupuregénérique » (l'équivalent d'une coupure-pub) et redynamisant le récit de l'épisode.

À la manière d'un lecteur modèle, nous pouvons imaginer un spectateur modèle à la manière de $\mathrm{Eco}^{8}$, le producteur imagine et façonne un spectateur modèle basé sur des compétences lectorales qui ne dépendent pas seulement du texte mais des conditions de réception de celui-ci (programmation régulière des épisodes, téléchargement, visionnage de DVD....9. Pour le spectateur, le générique livre des clefs interprétatives pour la compréhension de l'épisode et surtout de la série"

Cette identité de la série est supportée par la grille de programme (heures et jours de diffusion) mais aussi dans le cas d'une diffusion télé, la musique, le(s) décor(s), et les possibles narratifs de la série. À noter aussi que pour ancrer plus rapidement l'épisode, cette pratique n'utilise que des logos alphanumériques, non-figuratifs, où l'on ne retrouve que le titre de la série, sans référence plastique à la série. Dans ce cadre, ce sont les fonctions phatiques et référentielles qui dominent en suscitant la visibilité, la reconnaissance et le faire-savoir ce qu'est la série dans le flux télévisuel.

Bref, cela remanie le "genre générique », en s'affranchissant des conventions, mais cela remanie surtout sa fonction et l'identité de la série. Si l'identité visuelle et sonore se condense dans cette mini-séquence de trois secondes, elle se déporte surtout vers l'épisode. Ce qui sous-entend que la série possède une identité assez forte pour s'émanciper de son générique. Pour aller plus avant dans cette perspective, cette miniséquence peut se rapprocher du "carton », genre plus ancien, utilisé dans le cinéma muet, qui assure la fonction d'ancrage.

Dans le cas présent, cette mini-séquence assure une fonction de signature plus que d'introduction ou de description de la série.

Autre variation à rapprocher du logo, le mini-générique (pilote de la série Desperate Housewives ou Grey's Anatomy) reprend les codes visuels du générique sans narration, 
agrémenté d'une virgule sonore. Ce mini-générique est destiné à réaliser une transition entre deux scènes

\section{Le double générique}

11 Parallèlement à cet effacement du générique, Les 4400 en produisent un double. Le premier met en scène les protagonistes et leur quête de reconnaissance par l'humanité (la cause de leur venue), le deuxième s'articule sur les thèmes de la plasticité du temps, de l'espace et l'absence (une allégorie de fin du monde sans humain ?) ; ces deux parties de générique ne sont liées que sur une opposition sémiologique: présence humaine vs. absence d'humains.

\section{Écouter pour donner à voir}

Dernière innovation, la variation musicale au cours des épisodes de la saison: Weeds utilise depuis la deuxième saison une interprétation différente du titre "Little boxes »" ${ }^{10}$. Et pour continuer dans cette voie, la musique du générique de fin est toujours différente, en rapport étroit avec la scène finale. Cet intertexte musical est à rapprocher des images du générique : les images sont sur le thème du /même/ (en plus de la répétition du même générique), alors que la musique joue sur le registre de l'/altérité/ suscitant pour les fans une envie supplémentaire ${ }^{11}$ de regarder à chaque fois le générique. En rompant avec le code répétitif du générique, l'élément de surprise devient un gage de reconnaissance de l'identité de la série.

13 Pour résumer ces innovations, il se dégage des stratégies énonciatives de « dissimulation » (mini, logo, intégration), d'« occupation du territoire» (dédoublement) et "d'appétence» (hiatus musical et visuel). Ces nouvelles formes de générique s'orientent vers de nouveaux «besoins » de réception, en s'adaptant à un public de plus en plus exigeant et disponible.

\section{Interactions et ancrage}

\section{Le placement dans l'épisode et interactions}

14 Si tout se passe comme un jeu de poupées russes : générique, épisode, série, saison, inversement la syntagmatique change chaque série (fonction identitaire). Ainsi, qu'en estil de son placement dans l'épisode, et des interactions avec les autres unités de l'épisode? Voici sur le schéma suivant le résumé des situations possibles : 


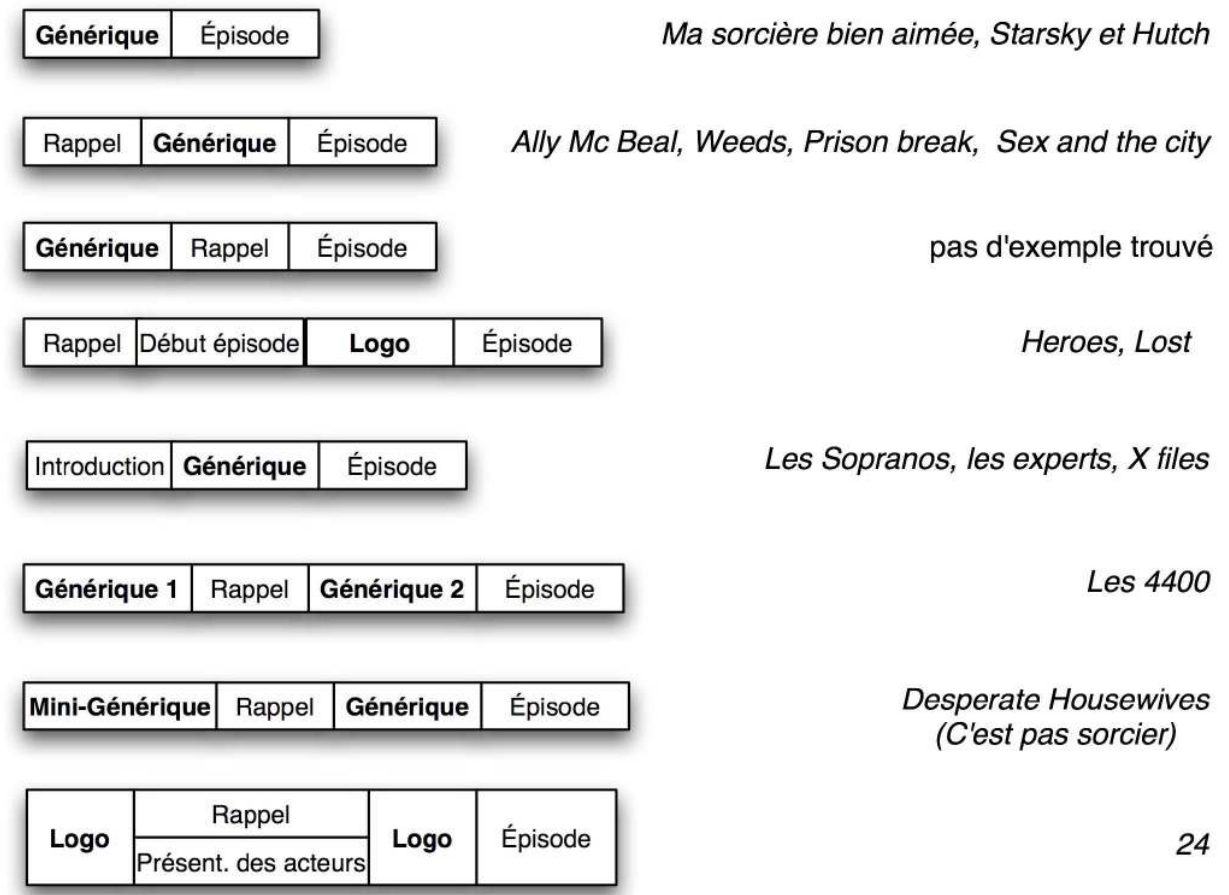

15 La cloison du générique n'est pas si étanche: elle agit sur l'épisode et ces interactions sont à rapprocher d'autres émissions, comme C'est pas Sorcier (situer l'action dans le camion/studio).

Intercalé avec ou entre les autres paratextes, il permet une pause dans la narration, à la suite d'un teasing et de relancer l'économie narrative: relance sur un autre aspect de l'épisode, mise en suspens à la suite d'une intrigue dans le pré-générique. Inversement 24 condense la présentation des acteurs (qui relève du générique) se superposant à l'introduction de l'épisode en utilisant un plan fixe (effet zoom), ici l'épisode s'invite dans le générique et créé l'identité de l'épisode et de la série.

objet souple s'adaptant au contexte de réception (générique en DVD ou mini-générique en programmation télévisuelle), le générique interagit aussi avec son contexte, contaminant le flux télévisuel dans un entrelacs composite avec musique du générique et vidéo du générique publicitaire (effet identitaire intertextuel ou « mashup » sur W9 et M6 ).

\section{L'ancrage géographique}

Si l'on se place dans une hypothèse énonciative, le générique s'adresse au "spectateur " extra-diégétique qui n'est pas encore le «narrataire» du récit. Les éléments géographiques jouent un effet de réel et préfigurent le lieu de l'action en s'inscrivant dans un embrayage énonciatif (un je-ici-maintenant).

Pour situer le rôle d'ancrage des génériques dans le projet d'une sémiotique des cultures, François Rastier ${ }^{12}$ rappelle que les langues développent des représentations d'espaces anthropiques : identitaire, proximal et distal qui fondent les pratiques sociales.

La production de génériques obéit à cette observation, et leur création se réalise avec la même force centripète : de l'éloigné au plus proche, de manière concentrique. Même Star Trek n'ira jamais se perdre, préférant patrouiller à la limite de la zone distale «Espace, 
frontière de l'infini vers laquelle voyage notre vaisseau... ». Les séries télé fonctionnent sur le mode proximal et surtout identitaire, c'est-à-dire au plus près des héros, de leurs actes et de leurs actions.

Le bateau, la mer ou les rivages de La croisière s'amuse contextualisent et participent à l'impression référentielle pour pallier aux studios et le générique est là pour cadrer les lieux de l'action, jouant sur l'identitaire de la série. Ainsi, autant une série exhibe des intérieurs ${ }^{13}$, autant un générique se doit de faire exister hors les murs, ancrer et maintenir le substrat de l'action des protagonistes ${ }^{14}$.

Le générique de Dallas, comme le rappelle Jean Mottet, ${ }^{15}$ est basé sur un montage de plans présentant les principaux lieux de l'action : puits de pétrole, terres agricoles, immeubles de la ville de Dallas et le ranch de Southfork. Lieux vus et revus pour ancrer la diégèse dans le monde naturel, reconnaissable et partagé par tous, référence à un déjà vu (e.g. : le Golden Gate de Los Angeles pour Charmed,) « les gratte-ciels de Dallas raccordent au voisinage, ou plus exactement la visibilité de la ville produit le voisinage ${ }^{16}$.Cette convergence de l'extérieur dans les intérieurs de studios trouve son paroxysme dans Les Soprano, avec le motif du road trip autoroutier du héros à New York qui le conduit jusqu'à la porte de sa maison, entre mobilité et fixité. Si les freeways, expressways, parkways sont des figures du rêve américain, ils conduisent et sont reliés au domicile ; cet espace du dehors cautionne un espace du dedans, lieu de vie pour localiser des héros et des problématiques du quotidien.

\section{Vers un ancrage inchoatif?}

Si Steve Austin est issu de préoccupations biotechnologiques des années 70, le récit de sa transformation est donné par le générique. Celui-ci le fait passer d'une chute à une reconstruction, voix off: "un homme tout juste vivant... Messieurs, nous pouvons le reconstruire, nous en avons la possibilité technique, nous sommes capables de donner naissance au premier homme bionique ", puis à son devenir, voix off : «Steve Austin deviendra cet homme, il sera supérieur à ce qu'il était avant l'accident " , trois phases pour comprendre ses capacités. Phase de sanction des compétences dans le schéma narratif, agrémentée d'images scientifico-médicales et de sons techniquement datés, notre héros sera doté de nouveaux pouvoirs, mais l'histoire de la série ne commencera qu'après sa sortie de son opération, table rase du passé pour "construire » et "forger » un personnage. Ainsi le générique livrerait les présupposés narratifs indispensables au déroulement de la performance qui fait le corps du récit-épisode. ${ }^{17}$.

Pour L'agence tous risques, le motif du complot («Accusés d'un vol qu'ils n'ont pas commis, n'ayant aucun moyen d'en faire la preuve, ils fuient sans cesse devant leurs poursuivants, pour subsister ils emploient leurs compétences...») est la raison de leur fuite. Leurs compétences et attributs sont illustrés à la manière d'un Mac-GyverWYSIWYG, (What You See Is What You Get), terminologie issue de la conception des interfaces homme/machine où les héros présentent de manière visible et reconnaissable leurs qualités (force, ruse, camouflage, séduction). Ici, la sémiotique greimassienne fonctionne à merveille : le récit du générique a pour fonction de faire passer un sujet virtuel (antérieur à l'acquisition de la compétence) qui devient sujet actualisé (acquisition de la compétence) qui devient sujet réalisé (performance qui permet la conjonction avec l'objet de valeur). Ainsi, la magie de ce type de générique est de rappeler les modalités entre héros et objet pour chaque épisode. 


\section{Conclusion : fonctions et fonctionnement du générique}

Regarder un épisode de série, comme la lecture de texte, consiste à prélever sélectivement des éléments micro- ou macrostructurels, à les transformer en indices signifiants et à effectuer une (ou des) hypothèse(s) de sens. Certes, si le générique participe a minima aux constructions interprétatives en "préparant» le spectateur, il fonctionne pour beaucoup de séries comme un jeu-de-base-Meccano interprétatif. Pour cette compréhension (ou pré-compréhension) il y a sans doute plusieurs fonctions du générique, certaines purement «identitaires » comme l'étiquette ou le nom propre (cf. phénomène logo), certaines plus "génériques" prédisposant au genre de la série (policière, médicale...) ${ }^{18}$, d'autres plus narratives (La petite maison (elliptique) dans la prairie), d'autres plus connotatives (mettre dans l'ambiance).

Ces fonctions relèvent aussi de l'énonciation, car elles mettent en place les conditions de la réception pour l'énonciataire (cf. lecteur-modèle) mais elles ont aussi un aspect rhétorique, manifestant l'« ethos » de l'énonciateur qui manifeste ainsi son " état d'âme » de narrateur (tragique, ironique, comique, ... cf. Les Envahisseurs).

Enfin, si les génériques appartiennent au paratexte, ils sont bien à l'interface, au point de jonction entre l'énonciation et l'énoncé, avec des possibilités de " patchwork » épisode/ série, créant une tension entre les deux; musique et tempo du montage permettant de souligner les aspects identitaires et de genre de la série.

\section{BIBLIOGRAPHIE}

ECO Umberto, 1985, Lector in Fabula, Grasset, Paris.

MOTTET Jean, 2005, Série télévisée et espace domestique : la télévision, la maison, le monde, L'Harmattan, Paris.

RASTIER François, 2001, «L'action et le sens pour une sémiotique des cultures » in Journal des anthropologues, $n^{\circ} .85-86$, mai 2001, pp. 183-219.

\section{NOTES}

1. Récompensé depuis 2000 par un Emmy Award, ou voire encore le nombre de compilations musicales, de blind-tests, de parodies et de sites qui proposent le visionnage de génériques

2. Effet involontaire, mention nécessaire en pleine guerre froide, ou encore nécessité après le débarquement des martiens d'Orson Welles?

3. indiquant que la série a bien commencé ?

4. Voir l'analyse de la boîte à images: http://laboiteaimages.hautetfort.com/ archive/2005/09/01/les-menageres-desesperees-passent-aux-aveux.html

5. cas rarissime 
6. Code semi-symbolique : le logo de début est noir sur fond blanc, blanc sur fond noir pour celui de la fin. Le dernier générique d'Urgences saison 13 a suivi cette tendance en mêlant logo et images de la série, reprise des anciens génériques, déjà très centrés autour du logo, et ce mélange figuratif/logo se perpétue

7. Et de temps de diffusion allongé pour les publicités? ou de disponibilité des cerveaux pour celles-ci?

8. 1985

9. Le lecteur modèle est un ensemble de conditions de succès ou de bonheur (felicity conditions) établies textuellement, qui doivent être satisfaites pour qu'un texte soit pleinement actualisé dans son contenu potentiel Eco, (1985:77)

10. Protest song sur les banlieues, initialement interprétée par Malvina Reynolds pour la première fois en 1962

11. Le cadeau Bonux audiovisuel?

12. 2001

13. et inversement les plans de coupe à l'intérieur de l'épisode sont là pour maintenir cet effet de réel (e.g. : Los Angeles pour Charmed ou Las Vegas, New York ou Miami pour les différentes déclinaisons des Experts)

14. oubliant même les deux flics dans Deux flics à Miami

15. 2005:83

16. ibid.

17. Cette même opération sera dédoublée avec la série Super Jamie, poussé par le succès rencontré par la série masculine, le générique est sans explication ni voix off, intertextualité s'interprétant par la mise en relation des codes établis par la série masculine

18. Tous les génériques ne permettent pas forcément de comprendre la série : les isotopies et connections interprétatives n'étant pas toujours réalisées comme dans True calling)

\section{RÉSUMÉS}

Imaginez un monde où Mac Gyver ne bricolerait pas, ne sauterait du parapet dès le premier pont venu, sans ses compétences, une série sans générique, et sans musique...

Une série fonctionne de pair avec son générique: placé à l'interface du flux télévisuel et de l'épisode, il établit le contrat de fiction entre héros et acteur. Objet d'identité visuelle, il constitue un discours sur la série, mais quelles sont les relations série/générique ? Si les outils de la sémiotique greimassienne standard fonctionnent à merveille : génèse du don du héros (Dead Zone ), devenir du héros (L'homme qui valait trois milliards), présentation des adjuvants (Alias), ou des opposants (Smallville), ancrage référentiel (La croisière s'amuse), les génériques observent quant à eux, des règles d'agencement énonciatifs singuliers (macro et micro unités) réalisés avec différents matériaux.

Alors que certains présentent une narration intertextuelle (Super Jammie vs. Steve Austin) d'autres pratiqueront une présentation extradiégétique (Desperate Housewives). Enfin, derrière l'évolution des nouveaux types de génériques, trois tendances identitaires sont à l'œuvre: intégration, dissimulation et surprise. 
AUTEUR

STÉPHANE MASSA-BIDAL

Groupe SEMEIA, Université Lyon II 